\title{
Estrés oxidativo en el paciente crítico
}

\author{
Max Andresen $\mathbf{H}^{\mathbf{1 a}}$, Tomás Regueira $\mathbf{H}^{\mathbf{1 b}}$, \\ Federico Leighton ${ }^{2}$.
}

\author{
Oxidative stress \\ in critically ill patients
}

Among critically ill patients, several physio-pathological processes such as global and local hypo-perfusion, hypoxia, endothelial injury and acidosis have been associated with the production and release of large amounts of reactive oxygen species (ROS) in a non regulated fashion. Although in physiologic conditions ROS influence intracellular processes and participate in the defense against infectious organism, in critically ill conditions they are associated with potential oxidative damage over cellular structures and with persistent activation of the inflammatory response. Mechanisms associated with oxidative damage are activation of the macrophage-monocyte system and neutrophils, ischemia-reperfusion events and intracellular ROS production. Endogenous compounds, mainly enzymes, and dietary components act as antioxidant. Several studies show that in critically ill patients increase levels of ROS or reduction of antioxidant levels are related to disease severity. In animal models of critical diseases, antioxidant therapy has shown to reduce mortality. Nevertheless, there are few studies in humans that only show improvements in hemodynamic variables, reduction in inflammatory mediators levels, decreases in oxidized compounds and that suggest a lower incidence of multiple organ failure (Rev Méd Chile 2006; 134: 649-56).

(Key w ords: Multiple organ failure; Oxidative stress; Reactive oxygen specias)

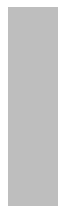

Recibido el 15 de julio, 2005. Aceptado el 29 de novien
1Unidad de Tratamiento Intensivo, Programa Medicin
2Laboratorio de Nutrición Molecular, Facultad de Cie
dad Católica de Chile, Santiago, Chile.
aMSSC Intensive Care
besidente Medicina Intensiva Universidad Católica

$\mathrm{D}$ iversos mecanismos fisiopatológicos participan en la génesis de la falla multiongánica (FOM) asociada a sepsis y shock. Estudios experimentales y clínicos han documentado que la respuesta inmune y la cascada inflamatoria pare-

Correspondencia a: Max Andresen Hernández. Unidad de Intensivo Médico, Hospital Clínico Universidad Católica de Chile. Marcoleta 367, Santiago. Fono: 56-2-3543703. E mail: andresen@med.puc.cl cen ser los elementos centrales en la génesis del daño celular y de órganos a nivel local y a distancia ${ }^{1}$. Algunos de estos mecanismos, como la hipoxia, la hipoperfusión, el daño endotelial y la activación celular, dan origen a grandes cantidades de radicales libres ${ }^{2}$ capaces de participar en la defensa frente a noxas infecciosas, pero también capaces de producir daño sobre estructuras celulares y tejidos, activando y perpetuando la respuesta inflamatoria. 


\section{ESTRÉS OXIDATIVO}

La producción de la energía necesaria para los diferentes procesos celulares requiere oxígeno, el cual es finalmente reducido a agua, luego de aceptar 4 electrones por acción del complejo citocromooxidasa de la cadena respiratoria mitocondrial. El oxígeno molecular (O2) posee dos electrones no apareados con espines paralelos, esto hace difícil que capte 2 electrones simultáneamente en las reacciones que interviene y explica la facilidad con que participa en reacciones univalentes. Normalmente, aproximadamente $2 \%$ del oxígeno es reducido en forma incompleta al aceptar un menor número de electrones, dando origen así a compuestos intermedios inestables, llamados especies reactivas de oxígeno (EROs) (Tabla 1). Estos son capaces de escapar de la mitocondria y producir daño sobre estructuras celulares, particularmente sobre membranas, proteínas, lípidos y ADN (Tabla 2).

El equilibrio de reducción-oxidación intracelular supone la presencia de compuestos antioxidantes enzimáticos y no enzimáticos capaces de destruir o neutralizar a estos radicales libres. El desbalance entre EROs y antioxidantes por la acción de alguna noxa, que estimule la producción de radicales libres o genere destrucción 0 consumo de los antioxidantes, se conoce como estrés oxidativo.

\section{MECANISMOS DE DAÑO}

Los mecanismos que explican el daño inducido por radicales libres son principalmente la activación del sistema monocito/macrófago y de neutrófilos, la activación mitocondrial intracelular y el mecanismo de isquemia y reperfusión.

El anión superóxido (O2.-) es producido por todas las células, sin embargo, en el paciente crítico se genera mayoritariamente en los polimorfonucleares activados ${ }^{3}$. Actúa como un agente proinflamatonio, siendo capaz de reclutar neutrófilos, inducir liberación de factores quimiotácticos y otros mediadores proinflamatorios, como TNF-alfa e IL-1, generar daño sobre ADN e iniciar peroxidación lipídica. Modula señales de transducción intracelular por la activación del factor nuclear (NF)-kB ${ }^{4}$ y heat shock factor-1. El peróxido de hidrógeno (H2O2) no posee electrones no apareados, sin embargo, se considera un EROs por su capacidad de inactivar enzimas, atravesar membranas celulares y reaccionar tanto con átomos de fierro $(\mathrm{Fe})$ como de cobre $(\mathrm{Cu})$ para producir $\mathrm{OH} \cdot$ a través de la reacción de Fenton.

El radical hidroxilo $(\mathrm{OH} \cdot)$ es un potente radical libre, varias miles de veces más reactivo que el O2.-. Produce daño principalmente sobre ADN, proteínas y lípidos, induciendo peroxidación lipídica y daño sobre membranas capaz de estimular la respuesta inflamatoria (Tabla 2).

\section{Tabla 1}

\begin{tabular}{|ll}
\hline Término & Definición \\
\hline Radicales libres & $\begin{array}{l}\text { Moléculas que contiene } 1 \text { o más electrones no pareados en la órbita } \\
\text { externa, capaz de reaccionar con otras moléculas y generar reaccio- } \\
\text { nes químicas que pueden conducir a daño molecular y celular. }\end{array}$ \\
$\begin{array}{l}\text { Especies reactivas de oxígeno } \\
\text { (EROs) }\end{array}$ & $\begin{array}{l}\text { Moléculas formadas por la reducción incompleta del oxígeno. Los } \\
\text { más estudiados son el anión superóxido (O2-.), el peróxido de hidró- } \\
\text { geno (H2O2), el radical hidroxilo (OH.) y el peroxinitrito (ONOO-). }\end{array}$ \\
Oxidación & Es la pérdida de electrones en una reacción química. \\
Reducción & Es la ganancia de electrones en una reacción química. \\
Equilibrio óxido-reducción & $\begin{array}{l}\text { Representa el balance en las reacciones de oxidación-reducción que } \\
\text { (REDOX) }\end{array}$ \\
Estrés oxidativo & $\begin{array}{l}\text { Correspon un medio. } \\
\text { acción de una noxa, que estimula la producción de radicales libres o }\end{array}$ \\
& genera destrucción o consumo de los antioxidantes. \\
Antioxidantes & Compuestos enzimáticos y no enzimáticos capaces de destruir o \\
neutralizar radicales libres evitando el daño oxidativo celular.
\end{tabular}


Tabla 2

\begin{tabular}{|ll|}
\hline Condición & Daño específico \\
\hline Daño sobre ADN & $\begin{array}{l}\text { Alteración de bases, cortes en una hebra, intercambio de cromátides } \\
\text { hermanas y entrecruzamientos de ADN y proteína. Esto conduce a } \\
\text { mutaciones y pérdidas en el control celular. }\end{array}$ \\
Peroxidación lipídica & $\begin{array}{l}\text { Reacción en cadena en la cual un OH. quita un átomo de hidrógeno de un } \\
\text { grupo metileno de un ácido grado de la membrana celular, transformán- } \\
\text { dolo en un nuevo radical libre (radical peroxilo). Este, a su vez, sustrae un } \\
\text { átomo de hidrógeno metilénico de un ácido grado vecino, proceso que se } \\
\text { repite en cadena hasta la muerte celular. } \\
\text { ROS alteran la conformación y la función de proteínas oxidando } \\
\text { aminoácidos específicos, como lisina, serina, arginina y prolina. La } \\
\text { pérdida de función frena procesos celulares e inducen la producción de } \\
\text { autoanticuerpos. } \\
\text { La alteración del balance REDOX cambia las señales intracelulares pudiendo } \\
\text { inducir proliferación, apoptosis, desregulación génica y necrosis. }\end{array}$ \\
\hline
\end{tabular}

El peroxinitrito se forma por la interacción entre 02.- y óxido nítrico. Perpetúa la acción del anión superóxido, actuando como agente proinflamatorio, inactiva la superóxido dismutasa $(\mathrm{SOD})^{5}$, induce peroxidación lipídica y produce depleción de glutatión ${ }^{6,7}$. Además, genera daño sobre ADN con depleción de nicotinamida adenina dinucleótido (NAD) y ATP. Su degradación no depende de una enzima específica, sino del ambiente químico celular; aunque su capacidad oxidativa puede ser bloqueada por antioxidantes como el glutatión y las vitaminas $\mathrm{C}$ y $\mathrm{E}^{8}$.

Los radicales libres de oxígeno no sólo participan en el daño celular por toxicidad directa, sino que también contribuyen a la hiporreactividad vascular resistente a vasopresores propia del shock séptico ${ }^{9-11}$.

La isquemia y reperfusión se asocia a la producción de radicales libres. Durante la isquemia, se consumen las reservas de ATP con formación de AMP, luego adenosina, inosina e hipoxantina y se induce también la enzima xantino oxidasa (XO) endotelial a partir de la xantino deshidrogenasa. Durante la reperfusión, con la llegada del oxígeno, la XO es capaz de transformar hipoxantina + 02 en xantina + ácido úrico + O2.-. En este escenario los antioxidantes endógenos, particularmente aquellos intracelulares, se consumen rápidamente. El ácido úrico se transfor- ma en un marcador de daño oxidativo por este mecanismo ${ }^{12}$.

\section{DAÑO SOBRE ESTRUCTURAS CELULARES}

La peroxidación lipídica es el daño oxidativo, producido particularmente por radical hidroxilo $(\mathrm{OH} \cdot)$, sobre los ácidos grasos poliinsaturados de las membranas celulares. Inicialmente, un ácido graso se oxida por salida de un átomo de hidrógeno de un grupo metileno hacía el $\mathrm{OH}$ - que actúa como agente oxidante, produciéndose radical peroxilo. Este, a su vez, repite el proceso en cadena hasta la muerte celular. El daño sobre lípidos puede ser medido a través de la detección de TBARS (del inglés thiobarbituric acid-reactive substances) en plasma u orina. Los TBARS podrían participar no sólo como marcadores de peroxidación lipídica, sino como inductores de FOM a distancia.

El daño sobre aminoácidos determina alteraciones en la conformación de la proteína y su función. La pérdida de función cambia o frena procesos celulares y puede, además, inducir la producción de autoanticuerpos. La oxidación proteica puede estimarse por carbonilos, que derivan de la oxidación directa de cadenas laterales de aminoácidos y de la ruptura oxidativa de proteínas. Los carbonilos 
son aparentemente difíciles de inducir y pueden, por lo tanto, indicar estrés oxidativo más severo. Existen estudios de la última década que asocian carbonilación a sepsis severa y SDRA ${ }^{13}$.

La metionina aparece como un nuevo marcador de daño oxidativo sobre proteínas. El primer paso en su oxidación genera metionina sulfóxido ${ }^{14}$, reversible por acción de reductasas (metionina sulfóxido reductasa); el segundo paso, que genera sulfonas, corresponde a una reacción irreversible. Numerosas proteínas y péptidos son oxidados en sus residuos de metionina, en algunas proteínas esto tiene poco o ningún efecto biológico, pero en otras puede llevar a inactivación. Los niveles de metionina sulfóxido han sido propuestos como un bio-marcador de estrés oxidativo in vivo ${ }^{15,16}$. La oxidación de residuos de metionina sin repercusión en procesos celulares y su reciclamiento por reductasas, pudiese corresponder a un mecanismo de protección frente al estrés oxidativo ${ }^{17}$.

\section{ANTIOXIDACIÓN}

La superóxido dismutasa (SOD) es una enzima intracelular distribuida en todo el organismo, capaz de transformar el O2.- en H2O2. Existen dos tipos, la intramitocondrial (SOD2) y la citoplasmática (SOD1); la primera es esencial para la vida, de hecho, los ratones SOD2 knockout no sobreviven ${ }^{18}$. La catalasa (CAT) es también un enzima intracelular, presente también en el interior de los glóbulos rojos, es capaz de transformar el $\mathrm{H} 2 \mathrm{O} 2$ en $\mathrm{OH} \cdot \mathrm{y}$ agua. El balance en la actividad de la CAT respecto de SOD es fundamental en mantener la homeostasis REDOX intracelular ${ }^{19}$. Glutatión peroxidasa es un complejo enzimático que contiene selenio y glutatión, está presente en el citoplasma y al interior de la mitocondria, participa activamente en la protección celular contra ROS, metabolizando $\mathrm{H} 2 \mathrm{O} 2$ y radicales peroxilo. Este sistema enzimático se recicla constantemente y la razón glutatión/glutatión disulfuro (reducido) permite estimar el grado de estrés oxidativo existente.

La vitamina C (ácido ascórbico) tiene funciones como cofactor enzimático. Su función antioxidante deriva de su capacidad de actuar como donante de electrones. Disminuye la peroxidación lipídica, los niveles de O2.-, H2O2 y del ión hipoclorito y mantiene estables los niveles de glutatión peroxidasa y de vitamina $E$, participando en su reciclamiento. La vitamina $\mathrm{C}$ se recicla a su vez desde ácido dehidroascórbico ${ }^{20}$. La vitamina E (alfa tocoferol) actúa unida a membrana, es el principal antioxidante capaz de prevenir y bloquear la peroxidación lipídica y se asocia a una respuesta inmune apropiada. La suplementación en pacientes con sepsis puede servir para modular la excesiva respuesta inflamatoria coordinada por los macrófagos, preservando la capacidad de respuesta a la infección ${ }^{21}$.

\section{ROL DEL ESTRÉS OXIDATIVO EN LOS ENFERMOS CRÍTICOS}

Diferentes trabajos han descrito estrés oxidativo en sepsis, en shock cardiogénico y distributivo, en el SDRA, en la coagulación intravascular diseminada (CID), en FOM, en pancreatitis, en quemados y asociado a fatiga diafragmática.

Vitamina E y C. Estudios en animales mostraron inicialmente que los niveles séricos y tisulares del alfa tocoferol (vitamina E), caen rápidamente en las primeras $24 \mathrm{~h}$ luego de inducir shock séptico por infusión de endotoxinas o por ligadura cecal y punción (LCP). Esto suginó que esta vitamina se consumía precozmente durante la sepsis, probablemente por participación en la inactivación de la peroxidación lipídica asociada a una elevada producción de ROS durante el shock séptico. Goode y $\operatorname{cols}^{22}$, en un estudio prospectivo con 16 pacientes en shock séptico y FOM, mostraron que los niveles de vitamina E, A y beta caroteno estaban significativamente disminuidos respecto de los controles sanos. En los pacientes con 30 más fallas orgánicas, los niveles de ácido tiobarbitúnico (TBARS), como marcador de peroxidación lipídica, estaban significativamente aumentados y se comelacionan en forma negativa con los niveles de vitaminas. Este grupo con 3 o más fallas incluyó sólo 5 pacientes. Sin embargo, los estudios en pacientes críticos con sepsis y SDRA han sido menos constantes en sus hallazgos respecto a vitamina $E$, aunque la mayońa muestra aumento en la peroxidación lipídica y consumo de alfa tocofe$\mathrm{rol}^{22-24}$, también existen estudios en que los niveles de vitamina E permanecen estables en el tiempo ${ }^{25-27}$, asociado a caída en los niveles de vitamina $\mathrm{C}$.

En nuestro estudio (Andresen M, Regueira T y cols. Temporary profile of Oxidation/Antioxidation in Septic Shock patients. Sometido a publicación), que comparó 21 pacientes en shock séptico con controles 
sanos, observamos que los niveles de vitamina $\mathrm{E}$ al ingreso fueron similares a los valores del grupo control y se mantuvieron estables en el tiempo, a pesar de existir clara evidencia de peroxidación lipídica al ingreso y que tendió a aumentar significativamente en el tiempo. Esto puede explicarse por un activo y persistente reciclamiento de vitamina $\mathrm{E}$ oxidada desde vitamina C. La regeneración de la vitamina E a partir del ácido ascórbico, sumado a la actividad antioxidante de esta última en el plasma, determina caída en los niveles plasmáticos de vitamina $C$, lo que se asocia a mayor gravedad ${ }^{28}$.

Capacidad antioxidante total y ácido úrico. La capacidad antioxidante total (CAT) refleja la capacidad global del plasma para prevenir el daño por radicales libres. El elevado consumo de antioxidantes presente en los enfermos críticos hace esperable que los niveles de CAT estuviesen disminuidos en estos enfermos, sin embargo, Pascual y cols ${ }^{29}$ encontraron que la CAT, en pacientes en shock séptico, no estaba disminuida respecto de los controles usando el método TRAP (Total Radical Peroxyl Trapping method). Observaron que existía un cambio en los componentes responsables de mantener la CAT estable, fundamentalmente por aumento de bilirrubina y ácido úrico plasmáticos. Los niveles de CAT caen a partir del tercer día, probablemente por mayor consumo de antioxidantes, tal vez en relación al aumento en la peroxidación lipídica, que no es compensado por un mayor aumento en los niveles de ácido úrico plasmático.

Los niveles de ácido úrico se elevan por activación de xantino oxidasa (XO) en tejidos sometidos a fenómenos de isquemia y reperfusión. Se elevan también los niveles plasmáticos de $\mathrm{XO}$, sin embargo, los pacientes más graves presentan niveles más bajos de XO plasmática y mayores niveles de lactato, lo que se explica porque estos pacientes conservan áreas de isquemia sin reperfundir y por tanto elevan lactato, pero no liberan XO al plasma ${ }^{30}$.

Sepsisy oxidación. La mayońa de los trabajos 22,23,25,31 concuerdan con nuestros resultados, en cuanto que durante la sepsis existe elevación de los niveles de oxidación lipídica, proteica y de $\mathrm{ADN}$, asociados a consumo concomitante de antioxidantes ${ }^{22}$. Winterbourn y cols ${ }^{31}$ mostraron en un estudio prospectivo en 22 pacientes con sepsis severa (APACHE 15-34) que los niveles de carbonilos ascendían precozmente tanto en plasma como en LBA, para luego caer en los siguientes días, manteniéndose sobre los valores de los controles. Metnitz y cols ${ }^{25}$ observaron que los pacientes con SDRA presentaban caída en los niveles de antioxidantes plasmáticos (vitamina $\mathrm{E}$ y $\mathrm{C}$, beta caroteno y selenio) y aumento de los productos de peroxidación lipídica. Otro estudio ${ }^{20}$, en enfermos críticos a los cuales se les midió alfa-tocoferol y TBARS, mostró que la concentración de alfa tocoferol fue más baja en pacientes críticos respecto de los controles y el promedio del TBARS fue significativamente más alto en pacientes de UCI. Los pacientes sépticos y de cirugía cardiaca presentaron también niveles más elevados que los controles, además los niveles de TBARS aumentaron significativamente luego de la aparición de coagulación intravascular diseminada (CID).

El proceso de destete de ventilación mecánica también se asocia a estrés oxidativo. Anzuelo y $\operatorname{cols}^{32}$, usando ratones depletados de vitamina E, observaron que durante la respiración contra resistencia, éstos presentaban una disminución en la capacidad contráctil del diafragma, aumento de la peroxidación lipídica y aumento en la activación de glutatión.

Aún no es claro cuáles marcadores de estrés oxidativo son los que mejor se correlacionan con la evolución clínica del paciente crítico. Sin embargo, en nuestra experiencia, los pacientes más graves evaluados por escalas de gravedad como el SOFA, con mayor compromiso hemodinámico, presentan mayores valores de daño oxidativo sobre lípidos y proteínas, mayores valores de vitamina $\mathrm{C}$ y de ácido úrico, todos los cuales aparecen como marcadores precoces y sensibles que pudiesen usarse individualmente o integrados como índice, para evaluar la intensidad del daño.

\section{TERAPIA ANTIOXIDANTE}

Estudios en animales con sepsis severa inducida, muestran que el aumento de los niveles de antioxidantes por intervención génica o por aporte exógeno, es capaz de reducir la mortalidad en forma significativa. El aporte exógeno de vitamina E, por ejemplo, ha mostrado disminuir la producción de TNF-alfa en respuesta a lipopolisacáridos endovenosos, asociado a disminución de mortalidad ${ }^{33}$.

Ritter y $\operatorname{cols}^{34}$ estudiaron ratones con sepsis inducida por LCP, y los dividieron en tres grupos: 
los controles, aquellos tratados con volumen y antibióticos (soporte básico), aquellos tratados con $\mathrm{N}$-acetil-cisteina (NAC) y un quelante de fierro (desferroxamina) (soporte antioxidante) y aquellos tratados con soporte básico más antioxidante. Observaron a las $12 \mathrm{~h}$, que los niveles de MPO y TBARS tisulares, como la producción de O2.mitocondrial, fueron significativamente menores en los ratones tratados con soporte antioxidante. El soporte antioxidante se asoció a una actividad de SOD y CAT más balanceada y la sobrevida del grupo con soporte básico y antioxidante fue significativamente mejor que el resto de los grupos.

Los estudios en humanos aún no han logrado mostrar beneficios en términos de sobrevida con el reemplazo antioxidante, sin embargo, se ha documentado que la terapia antioxidante efectivamente reduce los índices de productos oxidados, reduce los niveles de mediadores inflamatorios, mejora el índice cardíaco $^{35}$, aumenta la resistencia oxidativa de las moléculas de $\mathrm{LDL}^{36}$, mejora la oxigenación y disminuye los días de ventilación mecánica (VM) en $\mathrm{SDRA}^{37}$. Galley ${ }^{35}$ evaluó 30 pacientes con shock séptico a quienes randomizó para recibir NAC, vitamina C y E o dextrosa al 5\%. Observó que los pacientes que recibían suplementación antioxidante (16 pacientes) dejaban de elevar los niveles de peroxidación lipídica, mejoraban su índice cardíaco y presentaban una caída en la resistencia vascular sistémica. Nathens ${ }^{38}$, en un estudio multicéntrico, mostró que la suplementación con vitamina $\mathrm{C}$ y $\mathrm{E}$ en pacientes con trauma, disminuye la incidencia de FOM, los días de VM y de UCI y la incidencia de infecciones asociadas a VM. Otro estudio ${ }^{37}$ mostró que con la suplementación de vitamina E y B-caroteno en pacientes con SDRA se lograba mejorar la oxigenación y disminuir los días de ventilación mecánica (VM).

La suplementación de elementos traza, cofactores de enzimas antioxidantes (cobre, manganeso, zinc y selenio), ha mostrado resultados dispares ${ }^{39}$, sólo un estudio $^{40}$ mostró caída en la incidencia de FOM con el aporte exógeno de selenio. El selenio es un cofactor esencial para la funcionalidad de la glutatión peroxidasa y sus niveles plasmáticos también se han comelacionado con el grado de estrés oxidativo.

El glutatión es un tripéptido formado por glutamina, cisteína y glicina, participa en varios procesos biológicos como síntesis proteica, transporte transmembrana, metabolismo intermediario y maduración celular, entre otros, por lo que es esencial para la funcionalidad e integridad estructural celular en los tejidos y se le ha propuesto como un indicador más preciso de salud celular. La caída en los niveles plasmáticos de glutatión se asocia a muerte celular, hiporreactividad vascular, disfunción endotelial, aumento del estrés oxidativo en respuesta a $\mathrm{ROS}^{41}$ y a peor pronóstico. Los bajos niveles de glutatión a nivel mitocondrial, aparecen como el último factor determinante en la vulnerabilidad al ataque oxidativo. La NAC presenta una estructura muy similar al glutatión y actúa además como donante de grupos sulfidrilos. Se ha documentado su capacidad para regenerar los depósitos de glutatión, de disminuir los niveles de IL8 y de inhibir la activación de NF-kB en células mononucleares humanas durante la sepsis severa ${ }^{42,43}$.

Por otro lado, el aporte exógeno de enzimas antioxidantes o la intervención en los procesos que éstas regulan, pudiese tener un rol en el tratamiento de estos enfermos. Leff y cols ${ }^{44}$ observaron que los pacientes con shock séptico que desarrollaban SDRA presentaban niveles previos más altos de SOD y CAT, por lo que intervenir sobre estas enzimas podría asociarse a beneficios terapéuticos en estos pacientes.

La terapia antioxidante profiláctica en pacientes de alto riesgo no ha mostrado beneficios. Lassnigg y $\operatorname{cols}^{45}$ no encontraron ningún beneficio al suplementar con vitamina $\mathrm{E}$ a pacientes sometidos a circulación extracorpórea por by pass cardiopulmonar.

\section{CONCLUSIÓN}

La existencia de estrés oxidativo parece suficientemente documentada en los enfermos críticos. La aparente falta de beneficios de la terapia antioxidante en términos de sobrevida en estudios humanos, en contraposición con los resultados en estudios animales, pudiese explicarse por varias razones. Probablemente el estrés oxidativo juegue un rol beneficioso hasta cierto punto en la respuesta inflamatoria, induciendo proliferación celular, activación génica y apoptosis. Sin embargo, no está claro el momento ni el grado en el cual esta respuesta se vuelve inapropiada, por lo mismo nuevos trabajos son necesarios para establecer más claramente el perfil temporal de la respuesta oxidativa en los enfermos críticos, buscar índices que señalen la oportunidad de intervenir y buscar cuáles compuestos antioxidantes son los óptimos, en qué dosis y en qué momento. 


\section{REFERENCIAS}

1. Zeni F, FreEMAn B, NATANSON C. Anti-inflammatory therapies to treat sepsis and septic shock: A reassessment. Crit Care Med 1997; 25: 1095-100.

2. Taylor DE, Ghio AJ, Piantadosi CA. Reactive oxygen species produced by liver mitochondria of rats in sepsis. Arch Biochem Biophys 1995; 316: 70-6.

3. Szabo C, Cuzzocrea S, Zingarem B, O’Connor M, SAIZMAN AL Endothelial dysfunction in a rat model of endotoxic shock. Importance of the activation of poly (ADP-ribose) synthetase by peroxynitrite. J Clin Invest 1997; 100: 723-35.

4. Cuzzocrea S, Riey DP, Caputi AP, Salvemin D. Antioxidant therapy: a new pharmacological approach in shock, inflammation, and ischemia/reperfusion injury. Pharmacol Rev 2001; 53: 135-59.

5. YamakuRa $F$, TaKa $H$, Fujimura $T$, Murayama $K$. Inactivation of human manganese-superoxide dismutase by peroxynitrite is caused by exclusive nitration of tyrosine 34 to 3-nitrotyrosine. J Biol Chem 1998; 273: 14085-9.

6. Van Der Vlet A, Smith D, O’Neill CA, Kaur H, Darley-Usmar V, Cross CE ET al. Interactions of peroxynitrite with human plasma and its constituents: oxidative damage and antioxidant depletion. Biochem J 1994; 303: 295-301.

7. Cuzzocrea S, Zingarew B, Caputi AP. Role of peroxynitrite and poly (ADP-ribosyl) synthetase activation in cardiovascular derangement induced by zymosan in the rat. Life Sci 1998; 63: 923-33.

8. VATASSERY GT. Oxidation of vitamin E, vitamin C, and thiols in rat brain synaptosomes by peroxynitrite. Biochem Pharmacol 1996; 52: 579-86.

9. MacArthur H, Westfall TC, Riley DP, Misko TP, SALVEMINI D. Inactivation of catecholamines by superoxide gives new insights on the pathogenesis of septic shock. Proc Natl Acad Sci USA 2000; 97: 9753-8.

10. Salvemini D, Wang ZQ, Zweier JL, Samouilov A, MacArthur H, Misko TP et al. A nonpeptidyl mimic of superoxide dismutase with therapeutic activity in rats. Science 1999; 286: 304-6.

11. Salvemini D, Riey DP, Lennon PJ, Wang ZQ, Currie MG, MacArthur H et al. Protective effects of a superoxide dismutase mimetic and peroxynitrite decomposition catalysts in endotoxin-induced intestinal damage. Br J Pharmacol 1999; 127: 685-92.

12. Nishino T. The conversion of xanthine dehydrogenase to xanthine oxidase and the role of the enzyme in reperfusion injury. J Biochem 1994; 116: 1-6.

13. Dalie-Donne I, Giustarini D, Colombo R, Rossi $R$, Mirzani A. Protein carbonylation in human diseases. Trends Mol Med 2003; 9: 169-76.

14. Moskovitz J, Poston JM, Berlett B, Nosworthy N, Szczepanowski R, StadTMAn E. Identification and characterization of a putative active site for peptide methionine sulfoxide reductase (MsrA) and its substrate stereospecificity. J Biol Chem 2000; 275: 14167-72.

15. Mohri M, Reinach PS, Kanayama A, Shimizu M, Moskovitz J, Hisatsune T et al. Suppression of the TNFalpha-induced increase in IL-1alpha expression by hypochlorite in human corneal epithelial cells. Invest Ophthalmol Vis Sci 2002; 43: 3190-5.

16. Mashima R, Nakanishi-Ueda T, Yamamoto Y. Simultaneous determination of methionine sulfoxide and methionine in blood plasma using gas chromatography-mass spectrometry. Anal Biochem 2003; 313: 28-33.

17. Hoshi T, HeinemanN S. Regulation of cell function by methionine oxidation and reduction. J Physiol 2001; 531(Pt 1): 1-11.

18. Melov S, Coskun P, Patel M, Tuinstra R, Cottrell B, JUN AS ET AL. Mitochondrial disease in superoxide dismutase 2 mutant mice. Proc Natl Acad Sci USA 1999; 96: 846-51.

19. BAynE AC, SoHAL RS. Effects of superoxide dismutase/catalase mimetics on life span and oxidative stress resistance in the housefly, Musca domestica. Free Radic Biol Med 2002; 32: 1229-34.

20. Ho CT, CHAN AC. Regeneration of vitamin $\mathrm{E}$ in rat polymorphonuclear leucocytes. FEBS Lett 1992; 306: 269-72.

21. GA山 F. Vitamin C, vitamin E and immune response. J Nutr Biochem 2005; 16: 257.

22. Goode HF, Cowley HC, Walker BE, Howdie PD, WEBSTER NR. Decreased antioxidant status and increased lipid peroxidation in patients with septic shock and secondary organ dysfunction. Crit Care Med 1995; 23: 646-51.

23. Takeda K, Shimada Y, Amano M, Sakai T, Okada T, Yoshiya I. Plasma lipid peroxides and alphatocopherol in critically ill patients. Crit Care Med 1984; 12: 957-9.

24. Richard C, Lemonnier F, Thibault M. Vitamin E deficiency and lipoperoxidation during adult respiratory distress syndrome. Crit Care Med 1990; 18: 4-9. 
25. Metnitz PG, Bartens C, Fischer M, Fridrich $P$, Steltzer H, Druml W. Antioxidant status in patients with acute respiratory distress syndrome. Intensive Care Med 1999; 25: 180-5.

26. Cross CE, Forte T, Stocker R, Louie S, Yamamoto Y, AMES BN, FREI B. Oxidative stress and abnormal cholesterol metabolism in patients with adult respiratory distress syndrome. J Lab Clin Med 1990; 115: 396-404.

27. KELY FJ. Vitamin E supplementation in the critically ill patient: too narrow a view? Nutr Clin Pract 1994; 9: 141-5.

28. Schorah CJ, Downing C, Piripitsi A, Galuvan L, AlHazaa AH, Sanderson MJ et al. Total vitamin C, ascorbic acid, and dehydroascorbic acid concentrations in plasma of critically ill patients. Am J Clin Nutr 1996; 63: 760-5.

29. Pascual C, Karzai W, Meier-Hellmann A, Oberhoffer M, Horn A, Bredle D et Al. Total plasma antioxidant capacity is not always decreased in sepsis. Crit Care Med 1998; 26: 705-9.

30. GaLey HF, Davies MJ, Webster NR. Xanthine oxidase activity and free radical generation in patients with sepsis syndrome. Crit Care Med 1996; 24: 1649-53.

31. Winterbourn CC, Buss IH, Chan TP, Plank LD, Clark MA, Windsor JA. Protein carbonyl measurements show evidence of early oxidative stress in critically ill patients. Crit Care Med 2000; 28: 143-9.

32. Anzueto A, Andrade FH, Maxwell LC, Levine SM, LaWRENCE RA, Jenkinson SG. Diaphragmatic function after resistive breathing in vitamin E-deficient rats. J Appl Physiol 1993; 74: 267-71.

33. Bulger EM, Maier RV. An argument for vitamin E supplementation in the management of systemic inflammatory response syndrome. Shock 2003; 19: 99-103.

34. Ritter C, Andrades ME, Reinke A, Menna-Barreto S, Moreira JC, Dal-Pizzol F. Treatment with Nacetylcysteine plus deferoxamine protects rats against oxidative stress and improves survival in sepsis. Crit Care Med 2004; 32: 342-9.

35. Gauey HF, Howdie PD, Walker BE, Webster NR. The effects of intravenous antioxidants in patients with septic shock. Free Radic Biol Med 1997; 23: 768-74.

36. Preiser JC, Van Gossum A, Berre J, Vincent JL, CARPENTIER Y. Enteral feeding with a solution enriched with antioxidant vitamins $\mathrm{A}, \mathrm{C}$, and $\mathrm{E}$ enhances the resistance to oxidative stress. Crit Care Med 2000; 28: 3828-32.

37. Gadek JE, Demichele SJ, Karlstad MD, Pacht ER, Donahoe M, Albertson TE et al. Effect of enteral feeding with eicosapentaenoic acid, gamma-linolenic acid, and antioxidants in patients with acute respiratory distress syndrome. Enteral Nutrition in ARDS Study Group. Crit Care Med 1999; 27: 140920.

38. Nathens AB, NefF MJ, Jurkovich GJ, Klotz P, Farver $\mathrm{K}$, RUZINSKi JT ET AL. Randomized, prospective trial of antioxidant supplementation in critically ill surgical patients. Ann Surg 2002; 236: 814-22.

39. Berger MM, Shenkin A. Trace elements in trauma and burns. Curr Opin Clin Nutr Metab Care 1998; 1: 513-7.

40. Angstwurm MW, Schottdorf J, Schopohl J, GaertNER R. Selenium replacement in patients with severe systemic inflammatory response syndrome improves clinical outcome. Crit Care Med 1999; 27: 1807-13.

41. Cuzzocrea S, Zingaremi B, O’Connor M, Salzman AL, Szabo C. Effect of L-buthionine-(S,R)-sulphoximine, an inhibitor of gamma-glutamylcysteine synthetase on peroxynitrite- and endotoxic shock-induced vascular failure. Br J Pharmacol 1998; 123: 525-37.

42. Paterson RL, GaLey HF, Webster NR. The effect of $\mathrm{N}$-acetylcysteine on nuclear factor-kappa B activation, interleukin-6, interleukin-8, and intercellular adhesion molecule-1 expression in patients with sepsis. Crit Care Med 2003; 31: 2574-8.

43. Heler AR, Groth G, Heler SC, Breitkreutz R, Nebe T, Quintel M, Косн T. N-acetylcysteine reduces respiratory burst but augments neutrophil phagocytosis in intensive care unit patients. Crit Care Med 2001; 29: 272-6.

44. Leff JA, Parsons PE, Day CE, Taniguchi N, Jochum M, FRITZ H. Serum antioxidants as predictors of adult respiratory distress syndrome in patients with sepsis. Lancet 1993; 341: 777-80.

45. Lassnigg A, Punz A, Barker R, Keznickl P, Manhart $\mathrm{N}$, Roth E ET AL. Influence of intravenous vitamin E supplementation in cardiac surgery on oxidative stress: a double-blinded, randomized, controlled study. Br J Anaesth 2003; 90: 148-54. 